\title{
The human WW45 protein enhances MST1-mediated apoptosis in vivo
}

\author{
XUELAI LUO ${ }^{1 *}$, ZHAOMING LI $^{1}$, QUN YAN $^{1}$, XIAOLAN LI $^{1}$, DEDING TAO $^{1}$, JING WANG $^{2}$, YAN LENG $^{1}$, \\ KEVIN GARDNER ${ }^{3}$, SUSAN I.V. JUDGE ${ }^{4}$, QINGDI Q. $\mathrm{LI}^{3}$, JUNBO HU ${ }^{1}$ and JIANPING GONG ${ }^{1}$

\begin{abstract}
Departments of ${ }^{1}$ Surgery and Institute of Cancer Research, Tongji Hospital, ${ }^{2}$ Immunology, Tongji Medical College, Central China University of Science and Technology, Wuhan 430030, P.R. China; ${ }^{3}$ Center for Cancer Research, National Cancer Institute, National Institutes of Health, Bethesda, MD 20892; ${ }^{4}$ MS Center of Excellence-East, VA Maryland Health Care System, and Department of Neurology, University of Maryland School of Medicine, Baltimore, MD 21201, USA
\end{abstract}

Received October 20, 2008; Accepted December 23, 2008

DOI: 10.3892/ijmm_00000139

\begin{abstract}
Mammalian sterile 20-like kinase 1 (MST1) is a serine/threonine protein kinase that is activated in response to a variety of apoptotic stimuli and causes apoptosis when overexpressed in mammalian cells. The physiological regulation and cellular targets of MST1 are not well understood. Using a yeast two-hybrid system, we identified human WW45 (hWW45, also called hSav1) as an MST1-binding protein. The association between the two proteins was confirmed by immunofluorescence and co-immunoprecipitation, and hWW45 was present in both the cytoplasm and nucleus. When hWW45 alone was over-expressed, it weakly induced apoptosis. However, hWW45 augmented MST1-induced apoptosis when the two were co-expressed. Conversely, RNA interferencemediated depletion of endogenous hWW45 suppressed MST1induced apoptosis. These results indicate that hWW45 is required to enhance MST1-mediated apoptosis in vivo and thus is a critical player in an MST1-driven cell death signaling pathway.
\end{abstract}

\section{Introduction}

Mammalian sterile 20-like kinase 1 (MST1) is a serine/ threonine kinase belonging to the germinal center kinase group II (GCK II) sub-family of mitogen-activated protein kinases (MAPKs) (1-3). It comprises a Ste20-related kinase

Correspondence to: Dr Junbo Hu or Dr Jianping Gong, Department of Surgery, Tongji Hospital, Tongji Medical School, Central China University of Science and Technology, Wuhan 430030, P.R. China E-mail: jbhu@tjh.tjmu.edu.cn or jpgong@tjh.tjmu.edu.cn

Dr Qingdi Quentin Li, National Institutes of Health, Building 10, Room 11N234, Bethesda, MD 20892, USA

E-mail: liquenti@mail.nih.gov

*Contributed equally

Key words: hWW45, mammalian sterile 20-like kinase 1, apoptosis catalytic domain in the amino-terminus, and a non-catalytic tail that contains an auto-inhibitory and dimerization domain, and two functional nuclear export signals (amino acid residues 341-379 and 441-451) in the carboxy-terminus (4,5). MST1 also contains two caspase cleavage sites at DEMD326S and TMTD349G (6), just amino-terminal to an auto-inhibitory domain.

Caspase-mediated cleavage of MST1 occurs during apoptosis initiated by various apoptotic stimuli, including treatment with staurosporine, etoposide, okadaic acid, anti-tumor drugs, arsenite, UV light, the engagement of CD95/Fas, and serum starvation (5,7-12). Caspase-mediated cleavage regulates the cellular localization of MST1. MST1 was reported to be exclusively located in the cytoplasm, but the truncation of the two nuclear export signals by caspases allows its nuclear translocation (5). Following translocation into the nucleus, MST1 exerts its effects through nuclear proteins such as deathassociated protein 4 (DAP4) (13), histone H2B (14), and inhibitor of caspase-activated DNase (ICAD) (15) to cause chromatin condensation, DNA fragmentation, and consequently apoptosis. However, caspase-mediated cleavage alone is insufficient to activate MST1. Full activation of MST1 during apoptosis requires phosphorylation at two sites (6), both $\mathrm{T}^{183}$ and ${ }^{187}$, to induce its kinase activity. Phosphorylation at these sites may be further amplified by dimerization. Phosphorylation eventually leads to the activation of MST1 by caspases and the induction of apoptosis (16).

Genetic analyses conducted in Drosophila suggest that the MST1 homolog Hippo functions in a conserved tumor suppressor pathway. It encodes a Ste-20 family protein kinase that restricts cell proliferation and promotes apoptosis in conjunction with two other proteins, Salvador (Sav) and Warts (Wts), which apparently down-regulate cyclin E, Drosophila inhibitor of apoptosis protein 1 (DIAP1), and other proteins (17-20). A similar signaling pathway appears to be conserved in mammalian cells $(18,19)$. Furthermore, recent studies demonstrated that MST1 mediates oxidative stress-induced cell death in primary mammalian neurons by directly activating the FOXO family of transcription factors. MST1 phosphorylates FOXO proteins and promotes their nuclear translocation for inducing cell death in neurons (21). Thus, the kinase activity of 
MST1 plays important roles in diverse biological processes in mammalian cells. Nevertheless, little is known about the physiological regulation of MST1 and its other targets in mammalian cells.

In this report, we sought to identify candidate proteins that interact with MST1 by using a yeast two-hybrid system. We identified hWW45 as one such factor. hWW45 contains two WW domains and a coiled-coil region and its mRNA is ubiquitously expressed. It was reported that hWW45 is mutated in several human cancer cell lines and the ortholog of hWW45 in Drosophila, Salvador (Sav), promotes both cell cycle exit and apoptosis through modulation of cyclin E and DIAP. However, the cellular function of hWW45 in mammalian cells is not fully investigated. In this study, we demonstrate that hWW45 is one of the proteins that binds to MST1 and augments MST1-induced apoptosis.

\section{Materials and methods}

Reagents. Dulbecco's modified Eagle's medium and fetal bovine serum were purchased from Gibco. Monoclonal antibodies specific for the hemagglutinin (HA) and FLAG epitopes were purchased from Sigma-Aldrich (St. Louis, MO, USA). Anti-hWW45 monoclonal antibody was from Abnova (Taipei City, Taiwan, R.O.C.), and MST1 monoclonal antibody was from Cell Signaling Technology (USA). Horseradish peroxidase-conjugated secondary antibodies (anti-rabbit or anti-mouse IgG) and TRITC- and FITC-conjugated goat antibodies to mouse and rabbit $\mathrm{IgG}$ were purchased from Pierce (Rockford, IL, USA). An Annexin V and propidium iodide (Annexin V-PI) kit was from BD Biosciences (San Diego, CA, USA). Protein A Sepharose CL-4B was obtained from Amersham Biosciences (AB, Uppsala, Sweden). Other chemical reagents were purchased from Generay Biotech (Wuhan, P.R. China) or Tiangen Company (Wuhan, P.R. China), unless stated otherwise.

Plasmids. The full-length MST1 cDNA was isolated from a human fetal liver cDNA library and expressed as a FLAGtagged protein after subcloning into the plasmid pcDNA4/TO (Invitrogen, CA, USA). The hWW45 cDNA was also obtained from the human fetal liver cDNA library and was subcloned into pCMV-HA. The authenticity of all constructs was verified by sequencing prior to their use in the experiments.

Cell culture and transfection. Human embryonic kidney 293 (HEK-293), colon cancer SW480, NIH3T3, AGS, MCF-7, HeLa, and NPA cells were cultured in Dulbecco's modified Eagle's medium supplemented with $10 \%$ fetal bovine serum, $100 \mathrm{U} / \mathrm{ml}$ penicillin, and $0.1 \mathrm{mg} / \mathrm{ml}$ streptomycin, in an atmosphere of $10 \% \mathrm{CO}_{2}$ at $37^{\circ} \mathrm{C}$. Cells were seeded in six-well plates at a density of $3 \times 10^{5}$ to $8 \times 10^{5}$ cells per well $18-24 \mathrm{~h}$ before transfection. The transfections were performed using Lipofectamine (Invitrogen), as recommended by the manufacturer.

Small interfering RNA experiments. For small interfering RNA (siRNA)-mediated down-regulation of hWW45, the following three siRNA sequences were purchased from Ribobio Co. (Guangzhou, P.R. China): siBC020537_001 sense: 5'-
CCAUGAUCUCUUC CAAAGA dTdT, and antisense: 3'dTdTGGUACUAGAGAAGGUUUCU; siBC020537_002 sense: 5'-GAAUUUGGAACCUAUUAdTdT, antisense: 3'dTdTGGCUUAAACCUUGGAUAAU; siBC020537_003 sense: 5'-AAUUGGAGCAGAUUGUUAdTdT, and antisense: 3'-dTdTCUUAACCUCGUCUAACAAU. Cells were seeded in six-well plates and transfected with siRNA or control siRNA duplexes using Lipofectamine 2000 (Invitrogen) according to the manufacturer's recommendations. Cells were collected for Western blot analysis after at least 3 days in culture.

Yeast two-hybrid screen. Yeast two-hybrid screening was performed using the Matchmaker Two-Hybrid System 3 (Clontech, USA) according to the manufacturer's protocol. Briefly, the open reading frame of wild-type human MST1 was fused to the DNA-binding domain of yeast transcription factor GAL4 and used as bait after transformation into Saccharomyces cerevesiae strain AH109 yeast. To screen for proteins that interact with MST1, the AH109 strain carrying the expression vector was mated with $S$. cerevesiae strain Y187, which was transformed with the human fetal liver library fused to the transcription activating domain of GAL4. The two mating cultures were grown under high-stringency conditions. Positive clones were amplified; the plasmids were rescued, and the inserts were sequenced to identify the interacting partner.

Western blotting and immunoprecipitation. Cells were extracted into cold lysis buffer [50 mM Tris- $\mathrm{HCl}(\mathrm{pH} 7.5)$, $150 \mathrm{mM} \mathrm{NaCl}, 1 \mathrm{mM}$ EDTA, $1 \mathrm{mM} \mathrm{MgCl}_{2}, 0.5 \%$ Triton X100, phosphatase inhibitor mix ( $1 \mathrm{mM} \mathrm{NaF}, 1 \mathrm{mM} \mathrm{Na}_{3} \mathrm{VO}_{4}$, and $1 \mathrm{mM}$ ß-glycerol phosphate), and protease inhibitor mix ( $1 \mathrm{mM}$ PMSF, $2 \mu \mathrm{g} / \mathrm{ml}$ aprotinin, $1 \mu \mathrm{g} / \mathrm{ml}$ leupeptin, and $1 \mu \mathrm{g} / \mathrm{ml}$ pepstatin A)]. Cell lysates were clarified by centrifugation at $10,000 \mathrm{xg}$ for $20 \mathrm{~min}$. For immunoprecipitation, the lysates were incubated with $1 \mu \mathrm{g}$ of anti-hWW45, -MST1, or -FLAG antibody for $2 \mathrm{~h}$ at $4^{\circ} \mathrm{C}$ with rocking, followed by the addition of Protein A Sepharose beads for $4 \mathrm{~h}$ at $4^{\circ} \mathrm{C}$. Immunoprecipitates were washed six times with cold lysis buffer, suspended in $60 \mu 1$ of 1xSDS, and boiled for $5 \mathrm{~min}$ to dissociate the immunocomplexes from the beads. The supernatant was collected after centrifugation at 4,000 $\mu \mathrm{g}$ for $5 \mathrm{sec}$. The samples were resolved by SDS/PAGE and transferred to a PVDF membrane (Amersham Biosciences). The membrane was blocked in TBS-T buffer $(20 \mathrm{mM}$ Tris- $\mathrm{HCl}$, $\mathrm{pH} 7.5,150 \mathrm{mM} \mathrm{NaCl}$, and $0.05 \%$ Tween 20) containing $5 \%$ $(\mathrm{w} / \mathrm{v})$ non-fat milk, at room temperature $\left(22^{\circ} \mathrm{C}\right)$ for $1 \mathrm{~h}$. After blocking, the membrane was incubated with the primary antibody overnight at $4^{\circ} \mathrm{C}$, followed by incubation with horseradish peroxidase-conjugated anti-IgG for $1 \mathrm{~h}$ at room temperature. Peroxidase activity was detected using a SuperSignal West Femto Maximum Sensitivity Substrate Trial Kit (Pierce).

Immunofluorescence. HeLa cells were grown on chamber slides for $36 \mathrm{~h}$, washed with PBS, and then fixed with $4 \%$ formaldehyde in PBS for $10 \mathrm{~min}$. The cells were permeabilized in PBS containing $0.1 \%$ Triton X-100 for $10 \mathrm{~min}$ and blocked with $2 \%$ bovine serum albumin in PBS for $10 \mathrm{~min}$. They were then incubated with anti-hWW45 and -MST1 monoclonal antibodies $(1 \mu \mathrm{g} / \mathrm{ml})$ for $1 \mathrm{~h}$, followed by incubation with TRITC- 
A

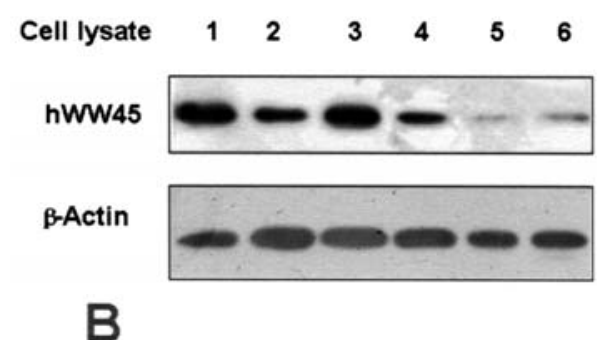

Figure 1. Expression and subcellular localization of hWW45 in human cells (A) Immunoblot analysis of hWW45 expression in various cell lines. Lanes 1-6, lysates from AGS, MCF-7, SW480, HeLa, NPA, and HEK-293 cells. (B) Subcellular localization of hWW45. Immunofluorescence shows the subcellular localization of hWW45 (green) and the nucleus (red) in HEK293 cells.
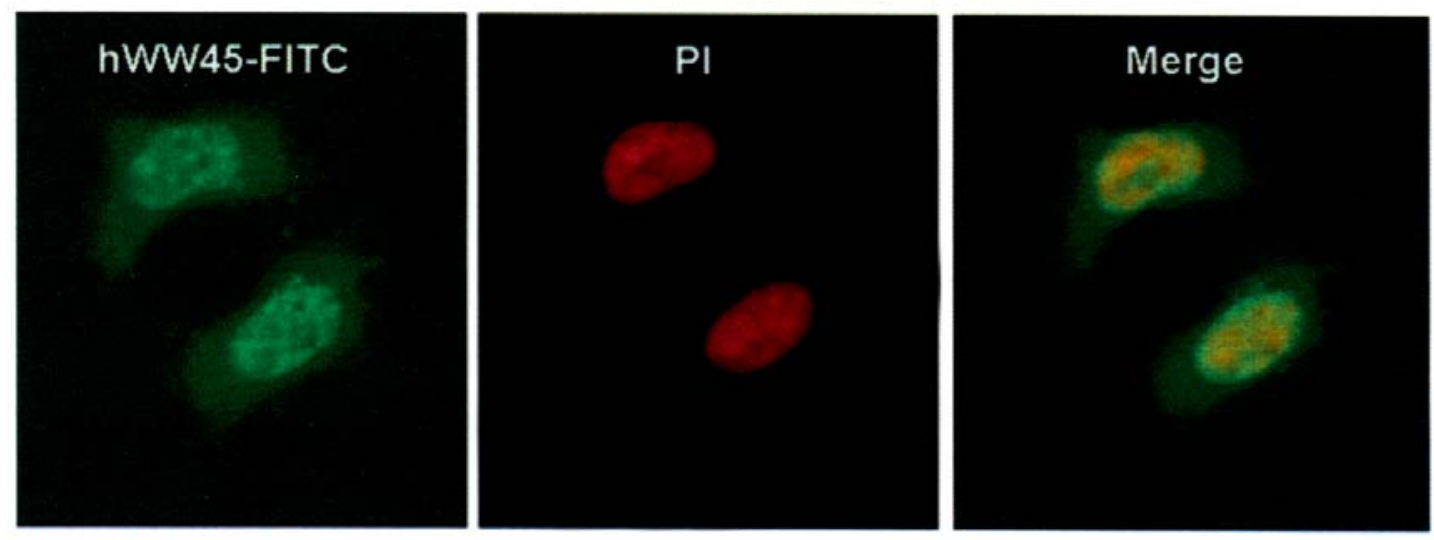

conjugated goat antibody to mouse IgG and FITC-conjugated goat antibody to rabbit IgG (1:100 dilution each) for $45 \mathrm{~min}$. Images were captured with a Leica confocal laser scanning microscope.

Cell apoptosis assay. At 24 to $48 \mathrm{~h}$ after transfection, the proapoptotic agent cisplatin was added at a final concentration of $50 \mu \mathrm{M}$, to increase the rate of apoptosis for $6 \mathrm{~h}$. Apoptotic cells were later quantified by flow cytometry using Annexin $\mathrm{V}$ and propidium iodide, as described previously (22). Briefly, cells were incubated with fluorescein isothiocyanate-conjugated Annexin V and subsequently analyzed using a Becton Dickinson FACStar Plus flow cytometer. The proportion of cells in a particular phase of the cell cycle was determined by CellQuest software.

Statistical analysis. Statistical differences between experimental groups were evaluated by Student's t-test. A value of $p<0.05$ was considered significant.

\section{Results}

hWW45 is ubiquitously expressed in human cell lines and localizes in both the cytoplasm and nucleus. We first examined the expression of hWW45 in human cell lines. Western blots of extracts prepared from a variety of human cell lines (Fig. 1A) showed the ubiquitous presence of a single major polypeptide band at $\sim 45 \mathrm{kDa}$, consistent with the size of hWW45. We next determined that hWW45 localizes in both the cytoplasm and nucleus, whether examined in HEK-293 (Fig. 1B) or HeLa cells (not shown). Thus, hWW45 is commonly expressed in human cell lines and localizes in both the cytoplasm and nucleus.

Identification of protein factors that interact with MST1 in a yeast two-hybrid screen. To identify MST1-interacting proteins, a yeast two-hybrid screen was performed on a human fetal liver
cDNA library, using full-length MST1 protein as bait. From a total of $1.23 \times 10^{6}$ clones screened under high-stringency conditions, 22 positive clones were identified. Three of these contained the full-length cDNA sequence of hWW45. We chose hWW45 for further experiments in this study. The others are being characterized separately.

To confirm the interaction between hWW45 and MST1, co-immunoprecipitation experiments were conducted in HEK-293 and HeLa cells. HEK-293 cells were transfected with an expression vector that expressed full-length MST1 as a FLAG-tagged protein, and anti-FLAG antibody was used to immunoprecipitate FLAG-tagged MST1. The presence of hWW45 protein in the immunoprecipitated complex was monitored by Western blot analysis with hWW45 antibodies. FLAG-tagged MST1 co-precipitated with hWW45, and antibodies to the endogenous hWW45 co-immunoprecipitated FLAG-MST1 protein (Fig. 2A). No hWW45 protein was found in immunoprecipitates from cells transfected with a control plasmid. We next investigated the endogenous interaction of hWW45 and MST1 in HeLa cells treated with the proapoptotic agent cisplatin $(50 \mu \mathrm{M})$ for $6 \mathrm{~h}$. When the endogenous MST1 was immunoprecipitated with an anti-MST1 antibody, hWW45 was also co-precipitated (Fig. 2B, upper). Reciprocally, when anti-hWW45 antibody was used to immunoprecipitate hWW45, Western blotting with anti-MST1 antibody revealed the co-precipitation of MST1 (Fig. 2B, lower). Further evidence of an in vivo interaction between MST1 and hWW45 was provided by an immunofluorescence analysis showing that a substantial proportion of MST1 in HeLa cells localized with hWW45 (Fig. 2C). These findings suggest that MST1 forms a complex with hWW45 in vivo.

hWW45 promotes MST1-mediated apoptosis in vivo. Finally, we examined whether hWW45 affects MST1-mediated apoptosis. As reported previously, MST1 over-expression is sufficient to induce apoptosis. HEK-293 cells were transfected 


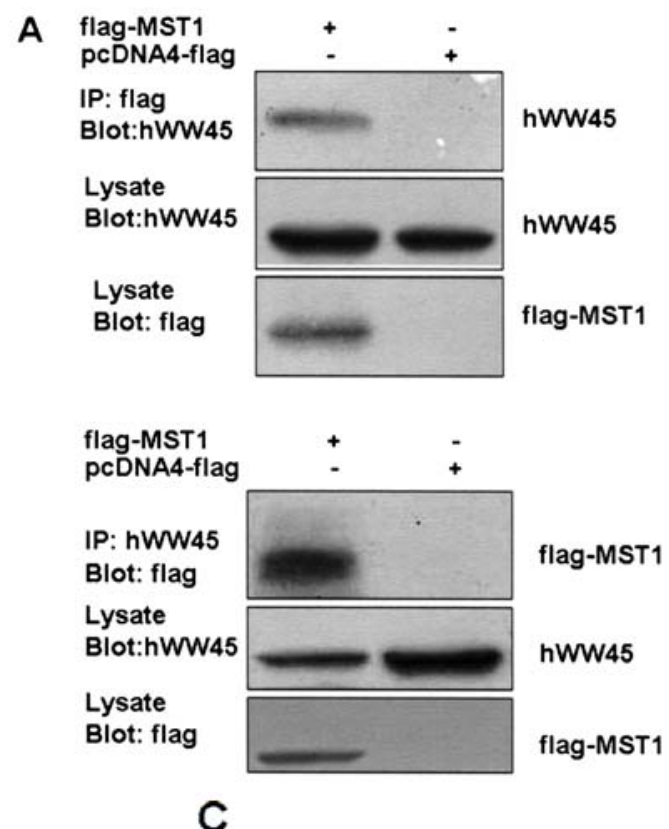

B
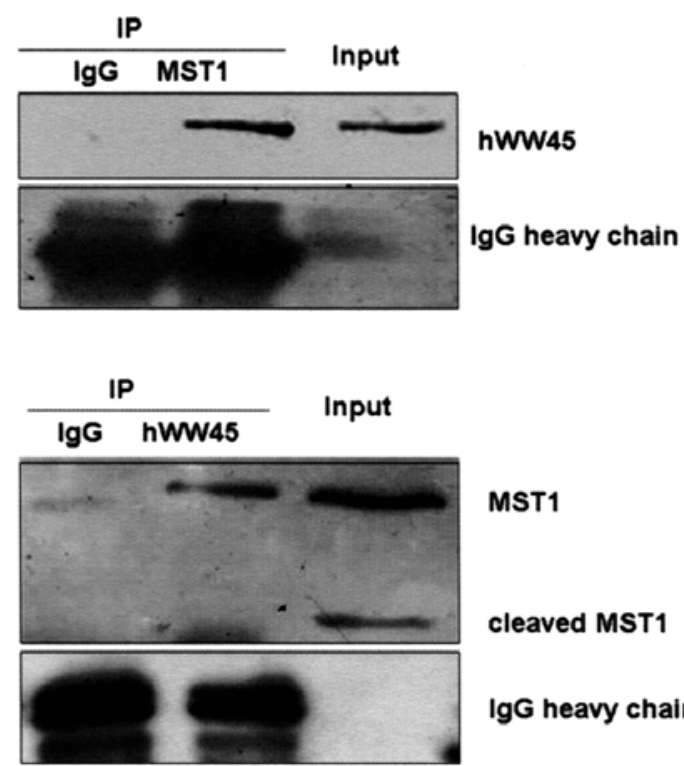

MST1

cleaved MST1

IgG heavy chain
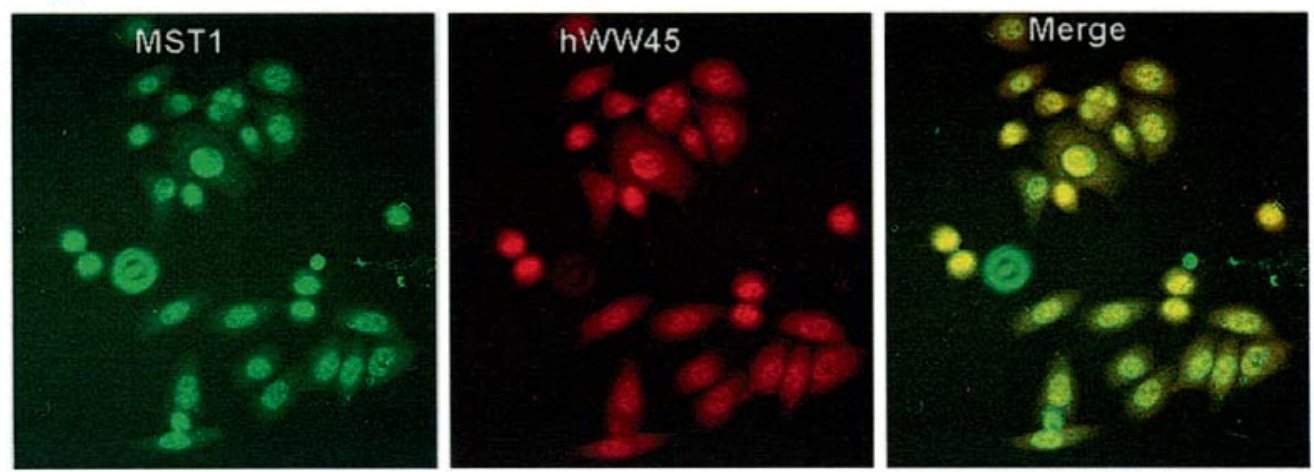

Figure 2. Association of hWW45 with MST1 in mammalian cells. (A) HEK-293 cells were transfected with empty FLAG vector or FLAG-MST1 vector. Cell lysates were extracted $36 \mathrm{~h}$ after transfection. Upper, the lysates were immunoprecipitated with anti-FLAG antibody and products were immunostained for hWW45 on a Western blot. Lower, the lysates were subjected to immunoprecipitation with anti-hWW45 antibody and products were immunostained for MST1 on a Western blot. (B) Endogenous hWW45 and MST1 proteins interact physiologically. Upper, HeLa cell extracts were immunoprecipitated with antibodies against MST1 (rabbit monoclonal antibody) or isotype-matched immunoglobulin G (IgG), and Western blots were probed with anti-hWW45 mouse monoclonal antibody. Lower, the reverse immunoprecipitation experiment with antibodies against hWW45 (mouse monoclonal antibody) or isotypematched IgG; Western blots were probed with anti-MST1 monoclonal antibody. IP, immuno-precipitation; IB, immunoblot; TL, total lysate. (C) Subcellular localization of hWW45 and MST1 in HeLa cells. Immunofluorescence shows subcellular localization of hWW45 (red) and MST1 (green). Co-localization of hWW45 and MST1 appears yellow after merging. Cells were visualized by confocal laser scanning microscopy.

with FLAG-MST1 and empty FLAG vectors. The pro-apoptotic agent cisplatin was added for $6 \mathrm{~h}$ to increase the rate of apoptosis. As shown in Fig. 3A, the incidence of apoptosis induced by MST1 was markedly increased compared with that in control cells (control FLAG vector versus FLAG-MST1: $12.6 \pm 3.0$ versus $24.5 \pm 2.4 ; \mathrm{p}<0.05$ ). Next, we over-expressed hWW45 in HEK-293 cells. The incidence of apoptosis was slightly higher in cells transfected with the HA-hWW45 vector than in cells transfected with the empty vector (Fig. 3B). However, the co-expression of hWW45 with a low dose of MST1 substantially increased the magnitude of MST1-induced apoptosis. The over-expression of MST1 alone induced 20\% apoptosis in HEK-293 cells and the apoptosis rate was significantly increased by co-expression of hWW45 with MST1 (24.5 \pm 2.4 versus 39.3 $\pm 4.0 ; \mathrm{p}<0.05$; Fig. 3C). Furthermore, following the knockdown of endogenous hWW45 by RNA interference (siRNA), the incidence of MST1-induced apoptosis in HEK-293 cells was markedly reduced, compared with that in control cells $(20.4 \pm 4.6$ versus $34.7 \pm 3.3 ; \mathrm{p}<0.05)$ (Fig. 3D). These findings suggest that hWW45 is required for MST1-mediated apoptosis in vivo.

\section{Discussion}

MST1, a ubiquitously expressed serine/threonine kinase, is activated by certain types of stress-inducers and participates in a wide range of apoptotic responses. To gain further insight into the mechanism of MST1 cellular actions, we used a yeast two-hybrid system to screen a fetal liver cDNA library. As a result, hWW45 was identified as a potential partner for MST1.

As a novel human WW domain gene, hWW45 encodes a protein of approximately $45 \mathrm{kD}$ comprising two WW domains and a coiled-coil region. hWW45 is ubiquitously expressed in adult tissues (23), and we confirmed this observation in various cell lines. Although the homolog of hWW45 in Drosophila, Salvador, was shown to promote both cell cycle exit and 
A
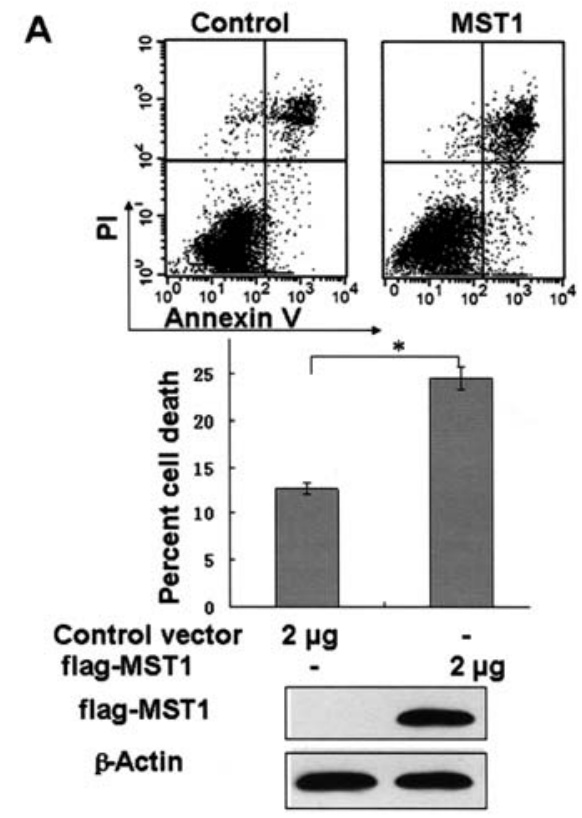

C Control MST1 hWW45 MST1+
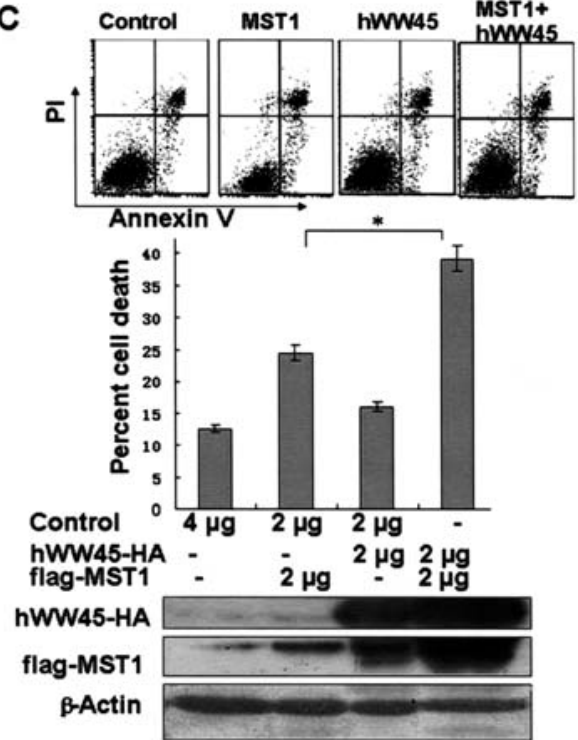

B
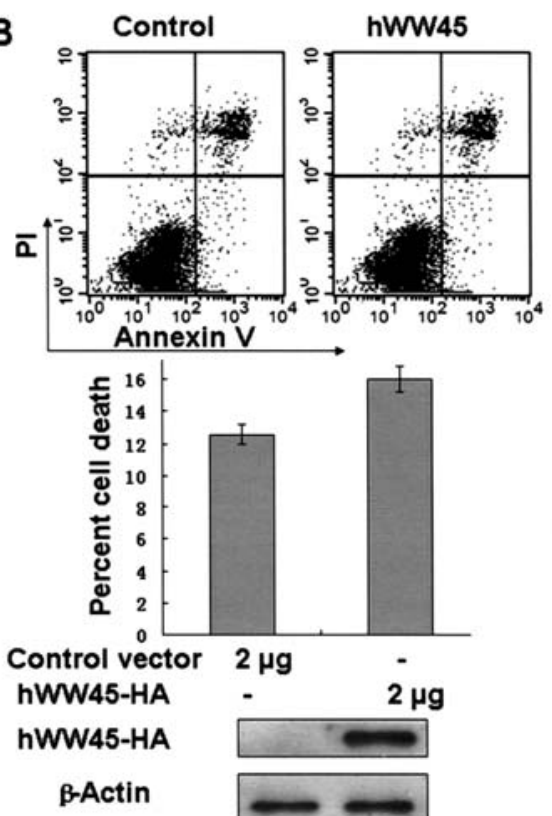

D

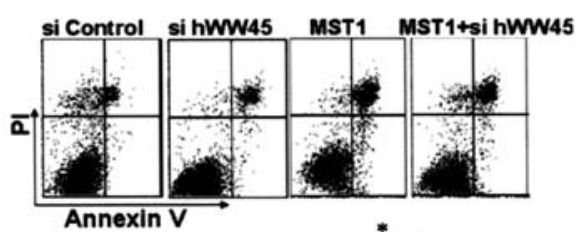

Annexin V
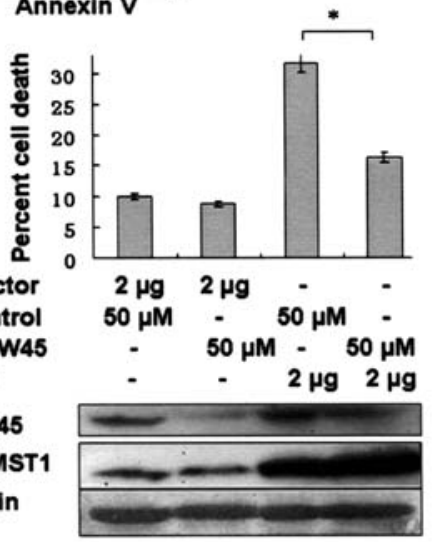

Control vector

SIRNA control

SIRNA hWW45

flag-MST1

hWW45

flag-MST1

B-Actin

Figure 3. Effect of hWW45 on MST1-induced apoptosis. (A) The effect of transiently transfected MST1 on cell death. HEK-293 cells were transfected with empty FLAG vector or FLAG-MST1 vector. The cells were incubated for $36 \mathrm{~h}$ and then cisplatin was added. After another $6 \mathrm{~h}$ of incubation, the cells were collected and analyzed for apoptosis by Annexin-V/PI. Cell lysates were subjected to immunoblot analysis with anti-FLAG antibody and with anti- 3 -actin as a loading control (bottom). (B) The effect of transient transfection with hWW45 on cell death. HEK-293 cells were transfected with empty HA vector or vector encoding HA-hWW45. After incubation, the cells were collected and analyzed for apoptosis as in A. (C) The effect of hWW45 over-expression on apoptosis induced by MST1. HEK-293 cells were transfected with plasmids encoding hWW45 and/or MST1 in the amounts indicated. After incubation, the cells were collected and analyzed for apoptosis as in A. The protein levels in the HEK-293 cells after transfection are shown at the bottom. (D) The effect of knocking down endogenous hWW45 via small interfering RNAs (siRNAs) on MST1-induced apoptosis. HEK-293 cells were transfected with siRNA duplexes capable of targeting the hWW45 and/or FLAG-MST1 plasmids, in the amounts indicated. After incubation, the cells were collected and analyzed for apoptosis as in A. Immunoblots for hWW45 and MST1 expression are shown at the bottom. ${ }^{*} \mathrm{p}<0.05$. All data are expressed as means \pm SEM.

apoptosis (17), the function of hWW45 in mammalian cells is not clear. We found that hWW45 binds to MST1 and colocalizes in both the cytoplasm and nucleus, which is consistent with previous studies showing that MST1 and hWW45 heterodimerize in an interaction requiring the conserved carboxyterminal coiled-coil domains of both proteins (24). Thus, hWW45 is a partner for MST1, and the two may act in a common apoptotic signaling pathway. Furthermore, the overexpression of hWW45 greatly enhanced the apoptotic efficacy of MST1, whereas the knockdown of endogenous hWW45 by RNA interference attenuated MST1-induced apoptosis in the present study. These results indicate that hWW45 promotes MST1-mediated apoptosis, although the underlying mechanism has yet to be elucidated.

Wu et al, reported a new pathway for cellular homeostasis that involves the scaffold protein Salvador (Sav) and two protein kinases, Hippo (Hpo) and Warts (Wts) (18). Both Hpo and Wts have human homologs, MST1/2 and LATS1/2, respectively. Hpo, together with Sav and $\mathrm{Wts}$, defines a signaling pathway that modulates both cell cycle and apoptosis. 
They suggested a model in which Sav acts as a scaffold and simultaneously binds to Hpo and Wts. This complex would allow Hpo to efficiently phosphorylate Wts, which apparently trigger cell cycle arrest and apoptosis by down-regulating cyclin E, DIAP1, and other targets $(18,19)$. The interaction between Hpo and Sav is mediated by their highly conserved carboxy-termini, while the interaction between Wts and Sav is mediated by the WW domains of Sav, which recognize a PPxY motif in Wts (18). A human homolog of Hpo was shown to rescue the overgrowth of Drosophila Hpo mutants, suggesting a high degree of functional conservation between flies and humans.

Recent studies demonstrated that hWW45 binds MST1 through the conserved carboxy-terminal coiled-coil domains of both proteins. LATS1/2 contains the PPXY motif, which could be recognized by the WW domains of hWW45. Thus, the role of hWW45 in the MST1 signaling pathway in human cells may resemble that of its Drosophila homolog Sav. In this model, hWW45 binds both MST1 and LATS through its coiled-coil domains and WW domains, respectively, and facilitates the phosphorylation of LATS by MST1. This model could explain the enhanced apoptotic efficacy of MST1 with the overexpression of hWW45 and the attenuated MST1-induced apoptosis with the down-regulation of endogenous hWW45. It also demonstrates the versatility of WW domain-containing proteins as platforms to link individual proteins into physiologically important networks $(25,26)$.

In summary, we identified hWW45 as an MST1-associated protein and showed that hWW45 localizes in both the cytoplasm and nucleus and is widely expressed in various cell lines. The over-expression of hWW45 initiates only slight apoptosis in HEK-293 cells but substantially augments the extent of apoptosis caused by MST1. hWW45 is likely to act as a platform to link MST1 and its related proteins, thereby facilitating the induction of apoptosis by MST1. This finding offers new insight into novel approaches for the study and treatment of proliferative diseases such as cancer.

\section{Acknowledgments}

This work was partly supported by grants from the Program for New Century Excellent Talents in Universities (NCET: No. 04-0699), the National Natural Science Foundation of China (NSFC: Nos. 30872472 and 30800569), and the National Basic Research Program of China (973: No. 2009CB521802).

\section{References}

1. Creasy CL and Chernoff J: Cloning and characterization of a human protein kinase with homology to Ste20. J Biol Chem 270: 21695-21700, 1995.

2. Taylor LK, Wang CR and Erikson RL: Newly identified stressresponsive protein kinases, Krs-1 and Krs-2. Proc Natl Acad Sci USA 93: 10099-10104, 1996.

3. Dan T, Watanabe NM and Kusumi A: The Ste20 group kinases as regulators of MAP kinase cascades. Trends Cell Biol 11: 220-230, 2001

4. Creasy CL, Ambrose D and Chernoff J: The Ste20-like protein kinase MST dimerizes and contains an inhibitory domain. J Biol Chem 271: 21049-21053, 1996.
5. Ura S, Masuyama N and Graves JD: Caspase cleavage of MST1 promotes nuclear translocation and chromatin condensation. Proc Natl Acad Sci USA 98: 10148-10153, 2001.

6. Graves JD, Draves KE and Gotohi Y: Both phosphorylation and caspase-mediated cleavage contribute to regulation of the Ste20like protein kinase MST1 during CD95/Fas-induced apoptosis. J Biol Chem 276: 14909-14915, 2001.

7. Kakeya H, Onose R and Osada H: Caspase-mediated activation of a 36-kDa myelin basic protein kinase during anticancer druginduced apoptosis. Cancer Res 58: 4888-4894, 1998.

8. Lee KK, Murakawa M and Nishida E: Proteolytic activation of MST/Krs, STE20-related protein kinase, by caspase during apoptosis. Oncogene 16: 3029-3037, 1998.

9. Reszka AA, Halasy-Nagy JM and Masarachia PJ: Bisphosphonates act directly on the osteoclast to induce caspase cleavage of MST1 kinase during apoptosis. A link between inhibition of the mevalonate pathway and regulation of an apoptosis-promoting kinase. J Biol Chem 274: 34967-34973, 1999.

10. Watabe $\mathrm{M}$, Kakeya $\mathrm{H}$ and Osada $\mathrm{H}$ : Requirement of protein kinase (Krs/MST) activation for MT-21-induced apoptosis. Oncogene 18: 5211-5220, 1999.

11. Watabe M, Kakeya H, Onose R and Osada H: Activation of $\mathrm{MST} / \mathrm{Krs}$ and c-Jun N-terminal kinases by different signaling pathways during cytotrienin A-induced apoptosis. J Biol Chem 275: 8766-8771, 2000.

12. Lu ML, Sato M and Cao B: UV irradiation-induced apoptosis leads to activation of a $36-\mathrm{kDa}$ myelin basic protein kinase in HL-60 cells. Proc Natl Acad Sci USA 93: 8977-8982, 1996.

13. Lin Y, Khokhlatchev A and Figeys D: Death-associated protein 4 binds MST1 and augments MST1-induced apoptosis. J Biol Chem 277: 47991-48001, 2002.

14. Cheung WL, Ajiro K and Samejima K: Apoptotic phosphorylation of histone $\mathrm{H} 2 \mathrm{~B}$ is mediated by mammalian sterile twenty kinase. Cell 113: 507-517, 2003.

15. Ura S, Masuyama N and Graves JD: MST1-JNK promotes apoptosis via caspase-dependent and -independent pathways. Genes Cells 6: 519-530, 2001

16. Glantschnig H, Rodan GA and Reszka AA: Mapping of MST1 kinase sites of phosphorylation, activation and autophosphorylation. J Biol Chem 277: 42987-42996, 2002.

17. Tapon N, Harvey KF and Bell DW: Salvador promotes both cell cycle exit and apoptosis in Drosophila and is mutated in human cancer cell lines. Cell 110: 467-478, 2002.

18. Wu S, Huang J and Dong J: Hippo encodes a Ste-20 family protein kinase that restricts cell proliferation and promotes apoptosis in conjunction with salvador and warts. Cell 114: 445-456, 2003.

19. Harvey KF, Pfleger CM and Hariharan IK: The Drosophila Mst ortholog, hippo, restricts growth and cell proliferation and promotes apoptosis. Cell 114: 457-467, 2003.

20. Hay BA and Guo M: Coupling cell growth, proliferation, and death. Hippo weighs in. Dev Cell 5: 361-363, 2003.

21. Lehtinen MK, Yuan K and Boag PR: A conserved MST-FOXO signaling pathway mediates oxidative-stress responses and extends life span. Cell 125: 987-1001, 2006.

22. Feng Y, Hu J, Li QQ and Gong J: Subcellular localization of caspase-3 activation correlates with changes in apoptotic morphology in MOLT-4 leukemia cells exposed to X-ray irradiation. Int J Oncol 27: 699-704, 2005.

23. Valverde P: Cloning, expression, and mapping of hWW45, a novel human WW domain-containing gene. Biochem Biophys Res Comm 276: 990-998, 2000.

24. Callus BA, Verhagen AM and Vaux DL: Association of mammalian sterile twenty kinases, Mst1 and Mst2, with hSalvador via C-terminal coiled-coil domains, leads to its stabilization and phosphorylation. FEBS J 273: 4264-4276, 2006.

25. Sudol M, Chen HI, Bougeret C, Einbond A and Bork P: Characterization of a novel protein-binding module-the WW domain. FEBS Lett 369: 67-71, 1995.

26. Ingham RJ, Colwill K and Howard C: WW domains provide a platform for the assembly of multiprotein networks. Mol Cell Biol 25: 7092-7106, 2005. 